\title{
Sodium Tanshinone IIA Sulfonate Enhances Effectiveness Rt-PA Treatment in Acute Ischemic Stroke Patients Associated with Ameliorating Blood-Brain Barrier Damage
}

\author{
Biying $\mathrm{Ji}^{1} \cdot$ Fei Zhou ${ }^{1} \cdot$ Lijuan Han ${ }^{1} \cdot$ Jun Yang ${ }^{2} \cdot$ Haijian Fan ${ }^{1} \cdot$ Shanshan $\mathrm{Li}^{1}$ • \\ Jingwei $\mathrm{Li}^{1} \cdot$ Xin $\mathrm{Zhang}^{1} \cdot$ Xiaoying Wang ${ }^{3} \cdot$ Xiangyan Chen $^{4} \cdot$ Yun Xu${ }^{1}$
}

Received: 16 November 2016/Revised: 11 February 2017 / Accepted: 13 February 2017 / Published online: 27 February 2017

(C) The Author(s) 2017. This article is published with open access at Springerlink.com

\begin{abstract}
Treatment with sodium tanshinone IIA sulfonate (STS) may ameliorate blood-brain barrier (BBB) damage in acute ischemic stroke patients receiving recombinant tissue plasminogen activator (rt-PA) thrombolysis and improve stroke patients' outcome. This randomized, single-center, placebo-controlled clinical trial investigated the potential effects and underlying mechanisms of STS. Forty-two acute ischemic stroke patients receiving intravenous rt-PA thrombolysis were randomized to intravenous administration either with STS (60 mg/day) $(n=21)$ or with equivalent volume of saline as a placebo $(n=21)$ after randomization for 10 days. Clinical outcomes, computer tomography perfusion (CTP) imaging with permeability-surface area product (PS) maps and serum levels of BBB damage biomarkers, were compared between the two groups. The percentage of patients with excellent functional outcome indicated by a 90 -day $\mathrm{mRS} \leq 1$ was significantly higher in the STS group than in the placebo group $(p=0.028)$. For patients with CTP imaging $(n=30)$,
\end{abstract}

Biying Ji, Fei Zhou, and Lijuan Han contributed equally to this article.

Yun Xu

xuyun20042001@aliyun.com

1 Department of Neurology and Radiology, Drum Tower Hospital, Medical School and The State Key Laboratory of Pharmaceutical Biotechnology and Jiangsu Key Laboratory for Molecular Medicine of Nanjing University, Nanjing, People's Republic of China

2 GE Healthcare, Shanghai, People's Republic of China

3 Departments of Neurology, Massachusetts General Hospital, Harvard Medical School, Charlestown, MA, USA

4 Departments of Medicine and Therapeutics, Chinese University of Hong Kong, Shatin, Hong Kong, SAR, China
PS in the ipsilateral lesion $(p=0.034)$ and relative PS $(p=0.013)$ were significantly lower in the STS group than that in placebo. STS-treated patients also had lower levels of matrix metalloproteinase (MMP)-9 $(p=0.036)$ and claudin-5 $(p=0.026)$, but higher levels of tissue inhibitor of metalloproteinase (TIMP)-1 $(p=0.040)$ than those in the placebo group. Post-stroke STS treatment could improve neurologic functional outcomes for acute ischemic stroke patients following rt-PA treatment by reducing BBB leakage and damage, which might be mechanistically associated with MMP-9 inhibition.

Keywords Blood-brain barrier · Sodium tanshinone IIA sulfonate $\cdot$ Thrombolysis $\cdot$ CT perfusion $\cdot$ Permeability surface

\section{Introduction}

Blood-brain barrier (BBB) breakdown is a major contributing factor to ischemic brain injury or hemorrhagic transformation (HT) and often leads to poor outcomes in acute ischemic stroke patients receiving recombinant tissue plasminogen activator (rtPA) treatment $[1,2]$. Tight-junction proteins such as claudin, junctional adhesion molecule, and occludin play essential roles in maintaining BBB integrity. Measurements of permeabilitysurface area product (PS), an indicator of BBB permeability, by computer tomography perfusion (CTP) imaging have been successfully applied for early identification of BBB damage and HT development in acute stroke patients [3, 4].

Sodium tanshinone IIA sulfonate (STS) is a water-soluble derivative of tanshinone IIA, a main bioactive component isolated from the roots of the Chinese herb Salviae miltiorrhiza Bunge (Danshen) [5]. STS has been widely used for treatments of cardiovascular and cerebrovascular diseases in China $[6,7]$. In mice with cerebral ischemia, STS could protect $\mathrm{BBB}$ and had a patent in China $[8,9]$. Here, we 
hypothesized that STS could work as a BBB protective agent that help acute ischemic stroke patients who received thrombolysis treatment recover better. In this study, we used CTPderived PS to reveal whether treatment with STS could reduce BBB leakage in acute ischemic stroke patients receiving rt-PA thrombolysis and sought to investigate the underlying mechanisms.

\section{Materials and Methods}

\section{Participants}

This single-centered, randomized, double-blinded prospective study was approved by the ethics committee of Drum Tower Hospital, Medical School of Nanjing University. An entry was made in the Chinese clinical trial registry (ChiCTR-ONRC-14004659). The inclusion criteria were as follows: (1) acute ischemic stroke patients receiving $\mathrm{rt}-\mathrm{PA}$ treatment, (2) at age between 18 to 80 years old, and (3) willing to participate in all followup neurologic and imaging examinations. We excluded patients with presence of platelet abnormalities (PLT $<100$ or $>300 \times 10^{9} / \mathrm{L}$ ), severe bleeding disorders, contraindications to iodinated contrast agent, a history of severe renal failure, or known or suspected infection. Informed consents were obtained from all participants involved in this study. Randomization sequences were computer generated. The flow chart of patient cohort selection is shown in Fig. 1.

\section{Treatments}

After rt-PA thrombolysis, STS (60 mg per day) was intravenously administrated daily to patients in the STS group for 10 days, while patients in the placebo group received equivalent administrations of saline. All participants received aspirin after thrombolysis $24 \mathrm{~h}$ for acute ischemic stroke.

\section{Neurologic Assessment}

Neurologic function outcomes were assessed using the National Institutes of Health Stroke Scale (NIHSS) score at $0,24 \mathrm{~h}$, and 10 days; activities of daily living (ADLs) score at $0 \mathrm{~h}$ and 10 days; and the modified Rankin Scale (mRS) at 90 days.

\section{CTP Scan Protocol and Image Analysis}

$\mathrm{CT}$ perfusion and non-contrast $\mathrm{CT}$ scans were performed on a 64-slice CT scanner (Discovery CT750 HD, GE Healthcare, Milwaukee, WI, USA). CTP started with intravenous injection of $50 \mathrm{~mL}$ of iodinated contrast agent $(350 \mathrm{mg} / \mathrm{mL}$,
Omnipaque, GE Healthcare, Shanghai, China) followed by a saline flush of $45 \mathrm{~mL}$ at $5 \mathrm{~mL} / \mathrm{s}$. CTP scans began after a delay of $5 \mathrm{~s}$ from contrast injection, and the following technical settings were applied: $80 \mathrm{kVp}, 150 \mathrm{mAs}$, temporal sampling rate of $2 \mathrm{~s}$ for $60 \mathrm{~s}$, and total axial coverage of $40 \mathrm{~mm}$ at $5-\mathrm{mm}$ slice thickness.

All CTP images were analyzed by experienced radiologists who were blinded to this research. For the PS maps and data, manually drawn region of interests (ROIs) in the ipsilateral hemisphere were compared to those in the contralateral hemisphere. PS values were calculated using CTP software (CT Kinetics, GE Healthcare, China). The corresponding ROIs for the contralateral side were generated automatically by mirroring the ipsilateral ROIs, and the relative PS (rPS) was defined as ipsilateral/ contralateral ROIs.

\section{Measurement of BBB Damage Biomarkers}

Venous blood were collected at $0,24 \mathrm{~h}$, and 10 days, and the separated serum was stored in aliquots at $-80{ }^{\circ} \mathrm{C}$ until biochemical analysis.

Serum levels of matrix metalloproteinase (MMP)-2 (R\&D, Minneapolis, IL, USA), MMP-9 (R\&D, Minneapolis, IL, USA), tissue inhibitor of metalloproteinase (TIMP)-1 (R\&D, Minneapolis, IL, USA), claudin-5 (CusaBio, Wu han, China), and zonula occluden (ZO)-1 (CusaBio, Wu han, China) were measured using commercially available ELISA kits according to the manufacturer's instructions.

\section{Study Outcomes}

The outcomes were the integrity of blood-brain barrier by measurement of PS, MMP-9, MMP-2, TIMP-1, claudin-5, and $\mathrm{ZO}-1$ and neurologic improvement by the score on NIHSS and the mRS at 90 days. Safety outcome measures the incidence of adverse event.

\section{Statistical Analysis}

All results were analyzed using SPSS (SPSS version 22.0, Chicago, Illinois, USA). The data were shown as mean \pm standard deviation (SD) or medians with interquartile ranges for continuous variables and proportions for categorical variables. Continuous variables were compared using $t$ tests, and categorical variables were analyzed using the Pearson $\chi^{2}$ test or Fisher's exact test. $P$ value $<0.05$ was considered statistically significant. 


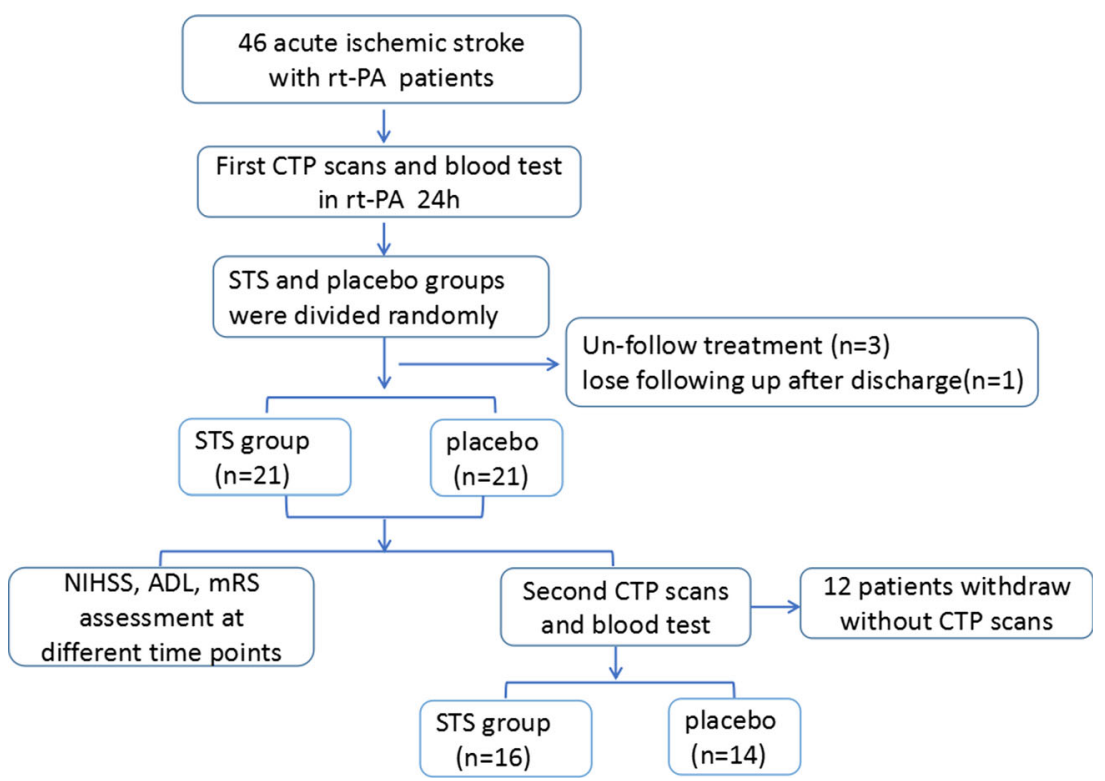

Fig. 1 Flow chart showing patient cohort selection in this study

\section{Results}

\section{Patient Characteristics}

From February 2014 to February 2016, 46 patients receiving rt-PA were included: 23 patients were treated with STS and 23 with placebo as control. Three patients had uncompleted treatment due to transferring to another department, and one patient was lost follow-up, thus leaving 21 patients in each group for clinical and prognostic analysis. General clinical characteristics (e.g., age, sex, risk factors, and NIHSS scores) and the distribution of baseline NIHSS at admission were similar (Table 1 and Fig. 2). In the 42 patients, 12 were unavailable to attend the second CT examinations, leaving 16 and 14 patients for imaging analysis in the STS and placebo groups, respectively.

Table 1 Patients characteristics

\begin{tabular}{lllll}
\hline & STS $(N=21)$ & Placebo $(N=21)$ & $\mathrm{F} / \chi^{2}$ & $P$ value \\
\hline Average age (years) & $63.81 \pm 9.872$ & $63.62 \pm 11.561$ & 1.321 & 0.954 \\
Male (\%) & $14(66.7 \%)$ & $14(66.7 \%)$ & 0.000 & 1.000 \\
Hypertension & $12(57.1 \%)$ & $17(81.0 \%)$ & 2.785 & 0.181 \\
Diabetes & $3(14.3 \%)$ & $5(23.8 \%)$ & 0.618 & 0.694 \\
Hyperlipidemia & $7(33.3 \%)$ & $6(28.6 \%)$ & 0.111 & 1.000 \\
Hyperhomocysteinemia & $5(23.8 \%)$ & $4(19.0 \%)$ & 0.141 & 1.000 \\
Atrial fibrillation & $4(19.0 \%)$ & $2(9.5 \%)$ & 0.778 & 0.663 \\
Coronary heart disease & $3(14.3 \%)$ & $1(4.8 \%)$ & 1.105 & 0.606 \\
Previous cerebral infarction & $2(9.5 \%)$ & $3(14.3 \%)$ & 0.227 & 1.000 \\
Smoking & $5(23.8 \%)$ & $3(14.3 \%)$ & 0.618 & 0.238 \\
Alcohol use & $6(28.6 \%)$ & $2(9.5 \%)$ & 2.471 & 0.238 \\
At admission & & & & \\
SBP (mmHg) & $153.48 \pm 17.665$ & $154.81 \pm 21.558$ & 1.896 & 0.828 \\
DBP (mmHg) & $86.62 \pm 14.333$ & $85.29 \pm 13.050$ & 0.527 & 0.754 \\
Glu (mmol/L) & $7.93 \pm 2.595$ & $8.66 \pm 4.070$ & 6.424 & 0.492 \\
Time to rt-PA (min) & $214.62 \pm 43.246$ & $198.71 \pm 42.717$ & 0.021 & 0.238 \\
NIHSS & $8.05 \pm 4.522$ & $7.38 \pm 5.220$ & 0.427 & 0.661 \\
ADL & $54.29 \pm 25.801$ & $53.33 \pm 27.034$ & 0.054 & 0.908 \\
\hline
\end{tabular}

$S B P$ systolic blood pressure, $D B P$ diastolic blood pressure 
Fig. 2 The distribution of baseline NIHSS between the two groups

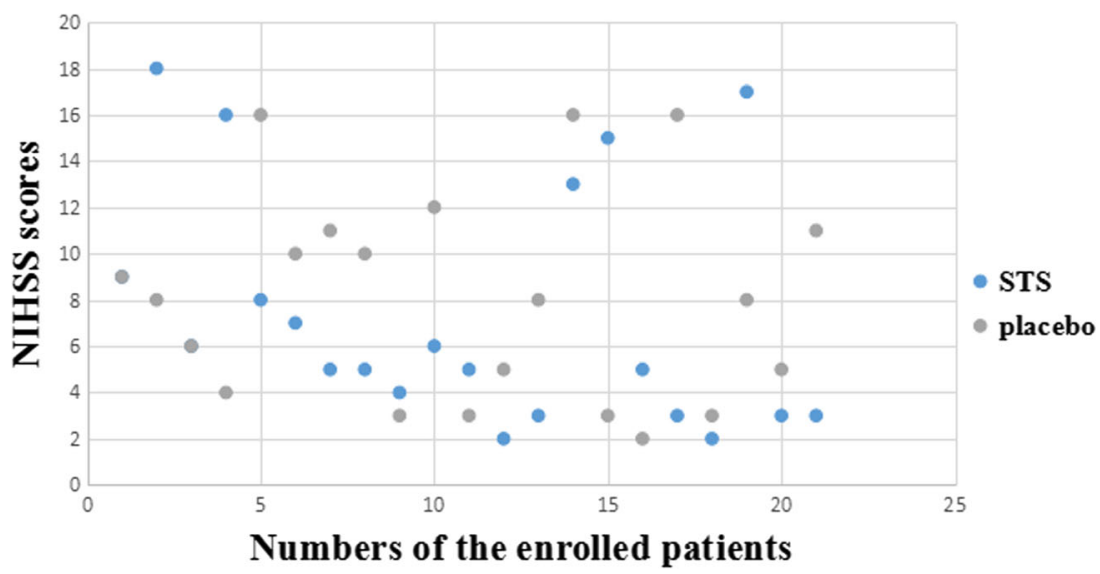

\section{Clinical Outcomes}

A battery of neurologic function assessments was used to investigate whether patients with acute ischemic stroke could benefit from STS after rt-PA thrombolysis. Results showed that in whole 42 patients (21 STS and 21 placebo), there were more patients with a 90-day mRS score $\leq 1$ (an excellent functional outcome) in the STS group than that in the placebo group. Though there is no statistical significance, STS did decrease the incidence of HT during hospitalization. No significant difference was found in the means of NIHSS and ADL score at 10 days after rt-PA treatment between the two groups (Table 2 and Fig. 3).

\section{Neuroimaging of BBB Permeability between STS and Placebo Groups}

CTP was performed to reveal the effects of STS treatment on BBB integrity. Twenty-four hours after rt-PA treatment, a baseline CTP was performed for each patient and there was no significant difference in BBB permeability (ipsilateral or contralateral and rPS) between the two groups. The follow-up CTP was performed at 10 days after the treatments. Moreover, the STS group showed significantly lower levels of ipsilateral PS and rPS than did the placebo group at 10 days (Table 3 and Fig. 4).

\section{Serum BBB Damage Biomarkers}

Serum levels of MMP-9, MMP-2, TIMP-1 and tight-junction proteins, including claudin-5 and ZO-1, in 30 patients (16 STS and 14 placebo) were measured to demonstrate BBB damage. The STS group had lower MMP-9 (633.352 vs $750.739 \mathrm{ng} / \mathrm{ml}$, $p=0.036$, Fig. 5a) and claudin-5 (337.822 vs $407.763 \mathrm{pg} / \mathrm{ml}$, $p=0.026$, Fig. 5b) levels but higher TIMP-1 expression (520.652 vs $459.567 \mathrm{ng} / \mathrm{ml}, p=0.040$, Fig. 5c) than did the placebo group at 10 days after thrombolytic therapy. However, at acute phase ( 0 and $24 \mathrm{~h})$, there were no significant differences in all biomarkers between the two groups.

\section{Discussion}

Current rt-PA thrombolytic therapy could augment BBB disruption in the acute stroke patients, which increase ischemic brain injury or HT [10]. Therefore, therapeutic strategies designed to alleviate BBB damage are needed to improve clinical outcomes of rt-PA thrombolysis [11]. Prognosis of rt-PAtreated patients with $\mathrm{mRS} \leq 1$ (an excellent functional outcome) was reported differently, from $42.7 \%$ to $54.6 \%$ at 3 months [12-14]. As Tsivgoulis reported, $\mathrm{mRS} \leq 1$ scores was $42.7 \%$, which is similar to our control group ( $\mathrm{mRS} \leq 1$ scores was $42.86 \%$ ). Effect of rt-PA therapy is affected by
Table 2 Patient clinical outcomes

\begin{tabular}{lllll}
\hline All enrolled patients & STS $(N=21)$ & Placebo $(N=21)$ & $\mathrm{F} / \chi^{2}$ & $P$ value \\
\hline 10 day-NIHSS & $2.81 \pm 2.64$ & $4.10 \pm 3.81$ & 1.853 & 0.211 \\
10 day-ADL & $82.62 \pm 20.89$ & $72.38 \pm 25.18$ & 1.016 & 0.159 \\
90 day-mRS $\leq 1$ & $16(76.19 \%)$ & $9(42.86 \%)$ & 6.462 & $0.028^{*}$ \\
HT during hospitalization & $2(9.52 \%)$ & $5(23.81 \%)$ & 1.543 & 0.410 \\
\hline
\end{tabular}

$* p<0.05$ 
Fig. 3 Distribution of $\mathrm{mRS}$ in the STS and placebo groups at 3 months after treatment. STS had more patients with 90-day $\mathrm{mRS}$ score $\leq 1$ in the whole 42 patients

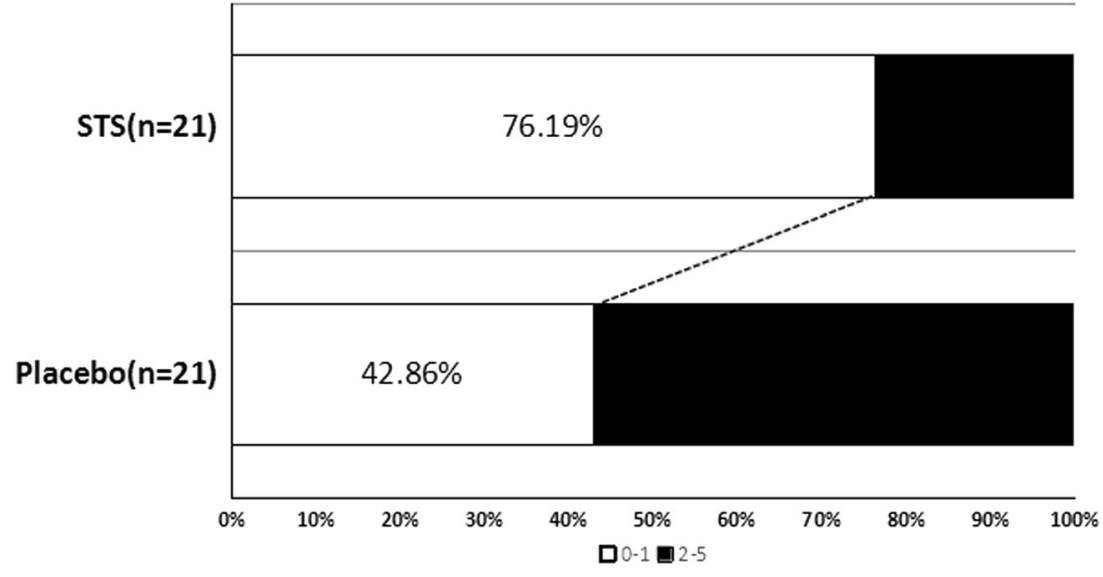

many factors, such as treatment time since stroke onset, age, stroke severity, BBB integrity, and so on. Our study found that there were more patients with a 90 -day $\mathrm{mRS}$ score $\leq 1$ in the STS group compared with the placebo group.

To investigate whether the neuroprotection of STS is associated with decreasing BBB disruption, PS was detected by dynamic contrast-enhanced CT [10, 15, 16]. Recently, CTP-derived PS quantification has been used to predict BBB permeability and HT for acute stroke patients because of its good reproducibility in hemodynamic measurement, wide accessibility, and relatively low cost $[2,4,10]$. The neuroimaging results from this study showed that STS treatment could reduce BBB-PS to ameliorate $\mathrm{BBB}$ damage and benefit clinical outcomes post-rtPA thrombolysis in acute stroke patients. Pathologically, release of tight junction adhesion molecules to blood circulation is associated with compromised BBB integrity in ischemic stroke [3]. Tanshinone IIA (precursor of STS) treatment has been previously shown to diminish BBB breakdown in experimental model of ischemic stroke [8, 9] and autoimmune encephalomyelitis [17]. Similarly, in this study, we found that STS treatment decreased the levels of BBB damage biomarkers, MMP-9 and claudin5 , in acute ischemic stroke patients after intravenous thrombolysis. From our observations, STS seemed to reduce the effects of BBB disruption by inhibiting MMP-9 activity and increasing expression of TIMP-1.

Next, we evaluated the infarct volumes and found no difference between the STS group $\left(2.020 \pm 1.762 \mathrm{~cm}^{3}\right)$ and the placebo group $\left(2.415 \pm 3.083 \mathrm{~cm}^{3}\right)$ after 10 days of treatment $(p=0.676)$. It suggested that STS did not reduce BBB damage through decreasing infarct volumes.

Furthermore, to study whether STS decreases cerebral hemorrhagic transformation, STS did reduce the trend of cerebral hemorrhagic transformation, but it was not significant, which may be correlated to the small sample research. STS could inhibit peripheral inflammatory cells into brain after stroke by suppressing BBB injury, which protects brain from immuno-inflammation and improves recover of stroke patients.

How STS protects the BBB from injury after stroke remains unclear. STS might improve BBB damage by
Table 3 BBB permeability measured by CTP-derived PS

\begin{tabular}{llll}
\hline Parameters & STS $(N=16)$ & Placebo $(N=14)$ & $P$ value \\
\hline Baseline & & & \\
$\quad$ Ipsilateral PS value $(\mathrm{ml} / 100 \mathrm{~g} / \mathrm{min})$ & $0.373 \pm 0.062$ & $0.395 \pm 0.073$ & 0.371 \\
Contralateral PS value $(\mathrm{ml} / 100 \mathrm{~g} / \mathrm{min})$ & $0.175 \pm 0.025$ & $0.179 \pm 0.022$ & 0.703 \\
rPS & $2.128 \pm 0.219$ & $2.225 \pm 0.380$ & 0.394 \\
PS region area $\left(\mathrm{cm}^{2}\right)$ & $2.586 \pm 2.461$ & $2.893 \pm 3.131$ & 0.766 \\
10 days after STS or placebo & & & \\
Ipsilateral PS value $(\mathrm{ml} / 100 \mathrm{~g} / \mathrm{min})$ & $0.266 \pm 0.083$ & $0.332 \pm 0.079$ & $0.034^{*}$ \\
Contralateral PS value $(\mathrm{ml} / 100 \mathrm{~g} / \mathrm{min})$ & $0.170 \pm 0.170$ & $0.172 \pm 0.017$ & 0.761 \\
rPS & $1.548 \pm 0.393$ & $1.910 \pm 0.345$ & $0.013^{*}$ \\
PS region area $\left(\mathrm{cm}^{2}\right)$ & $1.773 \pm 1.563$ & $2.287 \pm 2.214$ & 0.464 \\
Infarct volumes $\left(\mathrm{cm}^{3}\right)$ & $2.020 \pm 1.762$ & $2.415 \pm 3.083$ & 0.676 \\
\hline
\end{tabular}


Fig. 4 BBB-PS maps from the STS and placebo groups. Quantitative PS maps of both groups at baseline $(\mathbf{a}, \mathbf{c})$ and 10 days after STS or placebo treatment (b, d). At day 10, the patient with STS treatment showed a significant decline in BBB-PS when compared to the placebo

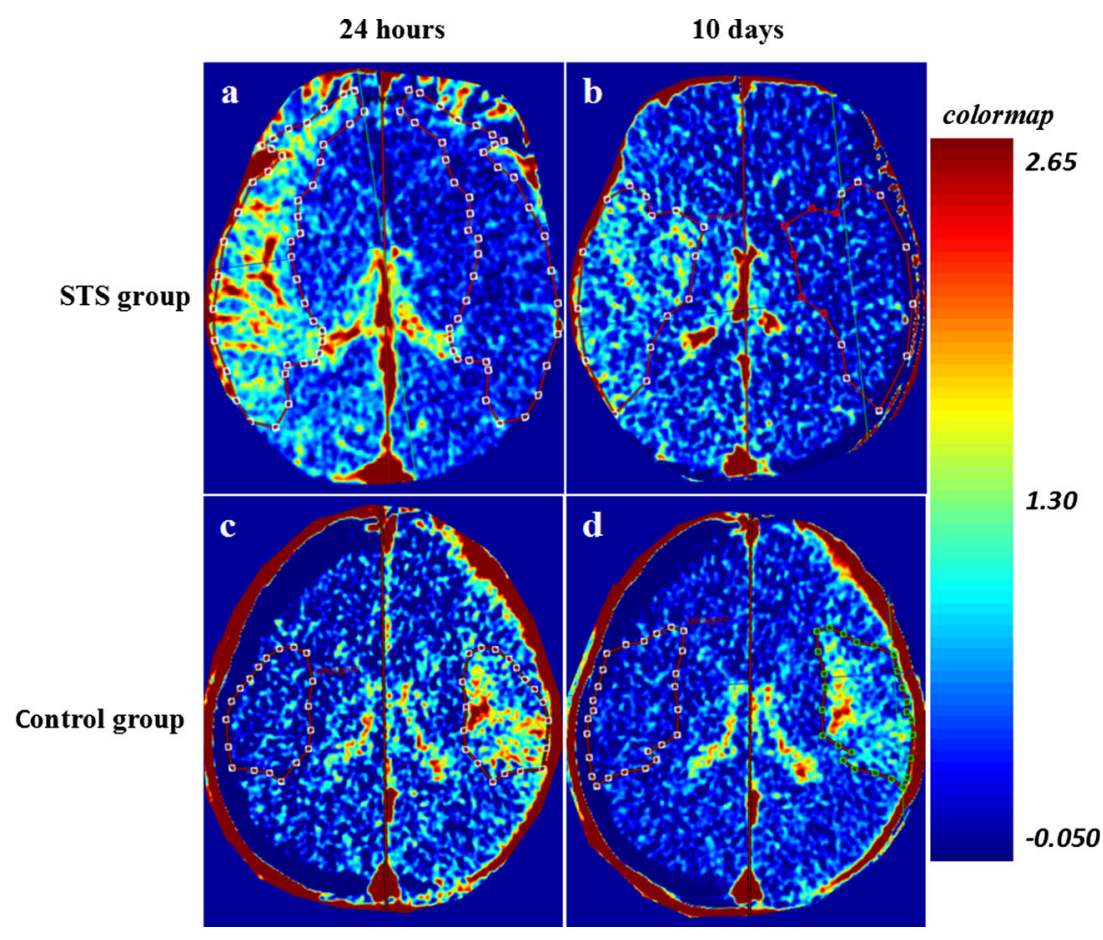

suppressing astrocyte-mediated inflammation or decreasing brain microvascular endothelial cell apoptosis after stroke.

Together, the neuroimaging and pathological evidence of our study could delineate the mechanistic pathway of STS treatment in improving BBB dysfunction post-rt-PA thrombolysis.

However, we had several limitations in the current work. First, to better elucidate the relation between BBB damage biomarkers and PS, MMPs and tight junction proteins obtained from cerebrospinal fluid (CSF) examination by lumbar puncture and a correlation study are needed in the future. Second, lack of BBB-PS measurements from CTP imaging at 90 days could not fully evaluate the effects of STS treatment on BBB repair. In addition, our study had a small sample size although the randomization has been applied. These questions will be addressed in our future work.
Fig. 5 Serum levels of BBB damage biomarkers. Serum MMP-9 (a), claudin-5 (b), and TIMP-1 (c) protein levels were measured at different time points using ELISA in the STS $(n=16)$ and placebo groups $(n=14)$. STS showed a lower MMP-9 and claudin-5 and higher TIMP-1 expression after 10 days treatment

\section{a}
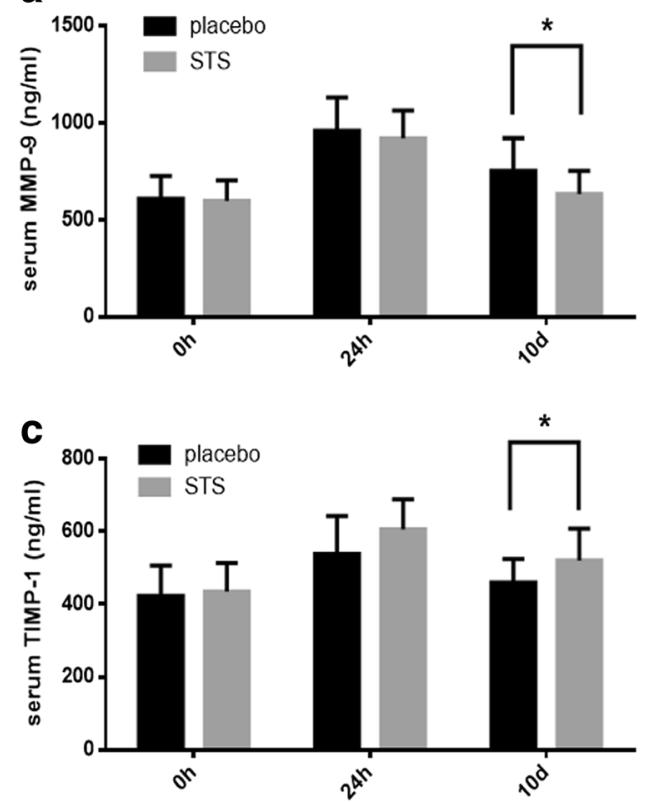

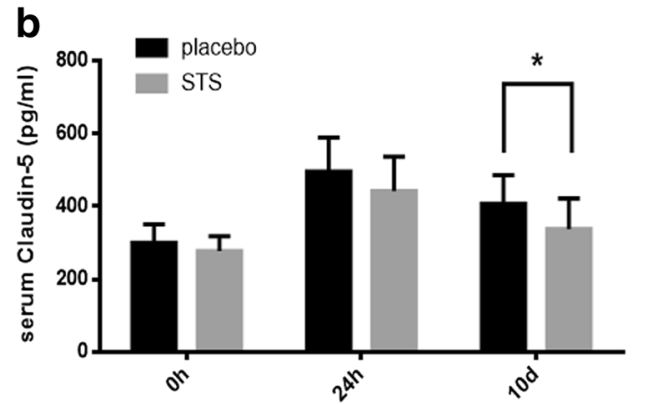


In conclusion, both neuroimaging and serum biomarkers of BBB damage in this study demonstrated that acute ischemic stroke patients might benefit from STS treatment by ameliorating/diminishing BBB disruption following rt-PA thrombolysis.

Acknowledgements $\mathrm{Xu}$ Yun designed the experiments and edited the manuscript. Ji Biying, Li Shanshan, Li Jingwei, and Zhang Xin collected patients and blood samples. Ji Biying performed ELISA test. Zhou Fei, Fan Haijian, and Yang Jun scanned and analyzed the CTP. Han Lijuan and Ji Biying wrote the manuscript. Wang Xiaoying and Chen Xiangyan revised this paper. All authors contributed to the data analysis and the manuscript preparation. We thank Brad Peterson for editing the English.

\section{Compliance with Ethical Standards}

Funding This research was supported by the National Natural Science Foundation of China $(81230026,81630028,81601016,81171085)$, the Natural Science Foundation (BE2016610, BK20160119) of Jiangsu Province, the National Key Research and Development Program of China (2016YFC1300500-504).

Conflict of Interest The authors declare that they have no conflict of interest.

Ethical Approval All procedures performed in studies involving human participants were in accordance with the ethical standards of the institutional and national research committee and with the 1964 Helsinki Declaration and its later amendments or comparable ethical standards.

Informed Consent Informed consent was obtained from all individual participants included in the study.

\section{Disclosures None.}

None of the authors are financially tied to the patent mentioned above.

Open Access This article is distributed under the terms of the Creative Commons Attribution 4.0 International License (http:// creativecommons.org/licenses/by/4.0/), which permits unrestricted use, distribution, and reproduction in any medium, provided you give appropriate credit to the original author(s) and the source, provide a link to the Creative Commons license, and indicate if changes were made.

\section{Reference}

1. Chaturvedi M, Kaczmarek L. Mmp-9 inhibition: a therapeutic strategy in ischemic stroke. Mol Neurobiol. 2014;49:563-73.

2. Horsch AD, Dankbaar JW, van Seeters T, et al. Relation between stroke severity, patient characteristics and ct-perfusion derived blood-brain barrier permeability measurements in acute ischemic stroke. Clin Neuroradiol. 2016;26:415-21.

3. Kazmierski R, Michalak S, Wencel-Warot A, et al. Serum tightjunction proteins predict hemorrhagic transformation in ischemic stroke patients. Neurology. 2012;79:1677-85.

4. Avsenik J, Bisdas S, Popovic KS. Blood-brain barrier permeability imaging using perfusion computed tomography. Radiol Oncol. 2014;49:107-14.

5. Tian $\mathrm{XH}, \mathrm{Wu} \mathrm{JH}$. Tanshinone derivatives: a patent review (January 2006- September 2012). Expert Opin Ther Pat. 2013;23:19-29.

6. Zhang $\mathrm{H}$, Long $\mathrm{M}, \mathrm{Wu} \mathrm{Z}$, et al. Sodium tanshinone IIA silate as an add-on therapy in patients with unstable angina pectoris. J Thorac Dis. 2014;6:1794-9.

7. Shang Q, Wang H, Li S, et al. The effect of sodium tanshinone IIA sulfate and simvastatin on elevated serum levels of inflammatory markers in patients with coronary heart disease: a study protocol for a randomized controlled trial. Evid Based Complement Alternat Med. 2013;2013:756519.

8. Zhang WJ, Feng J, Zhou R, et al. Tanshinone IIA protects the human blood-brain barrier model from leukocyte-associated hypoxia-reoxygenation injury. Eur J Pharmacol. 2010;648:146-52.

9. Wang L, Zhang X, Liu L, et al. Tanshinone ii a down-regulates hmgb1, rage, tlr4, nf-kappab expression, ameliorates BBB permeability and endothelial cell function, and protects rat brains against focal ischemia. Brain Res. 2010;1321:143-51.

10. Ozkul-Wermester O, Guegan-Massardier E, Triquenot A, et al. Increased blood-brain barrier permeability on perfusion computed tomography predicts hemorrhagic transformation in acute ischemic stroke. Eur Neurol. 2014;72:45-53.

11. Shi Y, Leak RK, Keep RF, et al. Translational stroke research on blood-brain barrier damage: challenges, perspectives, and goals. Transl Stroke Res. 2016;7:89-92.

12. Hacke W, Kaste M, Bluhmki E, et al. Thrombolysis with alteplase 3 to 4.5 hours after acute ischemic stroke. N Engl J Med. 2008;359: 1317-29.

13. Emberson J, Lees KR, Lyden P, et al. Effect of treatment delay, age, and stroke severity on the effects of intravenous thrombolysis with alteplase for acute ischaemic stroke: a meta-analysis of individual patient data from randomised trials. Lancet. 2014;384:1929-35.

14. Tsivgoulis G, Kadlecová P, Kobayashi A, et al. Safety of statin pretreatment in intravenous thrombolysis for acute ischemic stroke. Stroke. 2015;46:2681-4.

15. Ivanidze J, Kesavabhotla K, Kallas ON, et al. Evaluating bloodbrain barrier permeability in delayed cerebral infarction after aneurysmal subarachnoid hemorrhage. AJNR Am J Neuroradiol. 2015;36:850-4.

16. Neumann-Haefelin C, Brinker G, Uhlenkuken U, et al. Prediction of hemorrhagic transformation after thrombolytic therapy of clot embolism: an MRI investigation in rat brain. Stroke; J Cereb Circ. 2002;33:1392-8.

17. Yang X, Yan J, Feng J. Treatment with tanshinone iia suppresses disruption of the blood-brain barrier and reduces expression of adhesion molecules and chemokines in experimental autoimmune encephalomyelitis. Eur J Pharmacol. 2016;771:18-28. 